\title{
Influence of major BCR-ABL1 transcript subtype on outcome in patients with chronic myeloid leukemia in chronic phase treated frontline with nilotinib
}

\author{
Alexis Genthon ${ }^{1}$, Franck Emmanuel Nicolini2,3, Françoise Huguet ${ }^{3,4}$, Carole Colin- \\ Gil $^{4}$, Marc Berger ${ }^{3,5}$, Sandrine Saugues ${ }^{5}$, Alexandre Janel ${ }^{5}$, Sandrine Hayette ${ }^{3,6}$, \\ Pascale Cohny-Makhoul'3,7, Nadine Cadoux ${ }^{7}$, Jean-Michel Cayuela3,8, Lydia Campos99, \\ Denis Guyotat ${ }^{1,3}$ and Pascale Flandrin-Gresta ${ }^{3,9}$ \\ ${ }^{1}$ Service d'Hématologie et Thérapie Cellulaire, Institut Lucien Neuwirth, Saint-Priest-en-Jarez, France \\ ${ }^{2}$ Hematology Department, Centre Leon Berard, Lyon, France \\ ${ }^{3}$ French Group of CML, Bordeaux, France \\ ${ }^{4}$ Service d'Hématologie, Institut Universitaire du Cancer de Toulouse Oncopole, CHU de Toulouse, Toulouse, France \\ ${ }^{5}$ Service d'Hématologie Biologique, CHU Estaing and Université Clermont Auvergne, Clermont-Ferrand, France \\ ${ }^{6}$ Laboratoire d'Hématologie, Centre Hospitalier Lyon Sud, Pierre-Bénite, France \\ ${ }^{7}$ Hématologie Clinique, CH Annecy-Genevois, Epagny Metz-Tessy, France \\ ${ }^{8}$ Laboratoire de Biologie Moléculaire, Hôpital Saint-Louis, Paris, France \\ 'Laboratoire d'Hématologie, CHU de Saint-Etienne, Saint-Etienne, France \\ Correspondence to: Pascale Flandrin-Gresta, email: Pascale.flandrin-gresta@chu-st-etienne.fr \\ Keywords: CML; nilotinib; e13a2; e14a2; BCR-ABL1 \\ Received: January 15, $2019 \quad$ Accepted: June 05, $2020 \quad$ Published: June 30, 2020 \\ Copyright: Genthon et al. This is an open-access article distributed under the terms of the Creative Commons Attribution License 3.0 (CC \\ BY 3.0), which permits unrestricted use, distribution, and reproduction in any medium, provided the original author and source are credited.
}

\section{ABSTRACT}

Chronic myeloid leukemia (CML) is a myeloproliferative neoplasm characterized by the presence of $B C R-A B L 1$ transcript as a result of reciprocal translocation between chromosome 9 and 22. The most common transcripts subtypes are e13a2 (b2a2) and e14a2 (b3a2). The prognostic impact of the type of BCR-ABL1 transcript has been the subject of controversies over time. In the imatinib era, several studies have suggested a deeper and faster response in patients expressing e14a2. However, the impact on response after first line therapy with a second-generation tyrosine kinase inhibitor, nilotinib, is unknown.

We retrospectively evaluated 118 patients newly diagnosed with chronic phase CML and treated frontline with nilotinib inside or outside clinical trial in five French centers. Only patients expressing e14a2 or e13a2 transcripts alone were analyzed.

At baseline, 55.3\% expressed e14a2, 44.7\% expressed e13a2. The median age was 51 years and median follow-up was 49 months. Relative risks of CML at diagnosis were similar according to the ELTS score $(p=.87)$. Complete hematological response and complete cytogenetic response rates were similar among groups. Patients expressing e14a2 transcripts compared to e13a2 transcripts had deeper and faster molecular responses, when considering MMR $(100 \%$ vs $84.1 \%, p=.007)$ with a median time of 6.7 and 17.1 months or $M^{4.5}(100 \%$ vs $59.9 \%, p=.005)$ with a median time of $\mathbf{3 9 . 7}$ and $\mathbf{7 0 . 9}$ months, respectively. A sustained treatment free remission was observed in $10 / 10$ patients with e14a2 versus $1 / 3$ with e13a2 transcript $(p=.04)$.

In conclusion, even treated with nilotinib first line, patients with chronic phase CML expressing BCR-ABL1 e13a2 transcript have a lower rate of deep molecular responses. 


\section{INTRODUCTION}

Chronic myeloid leukemia (CML) is a myeloproliferative neoplasia characterized in chronic phase by increases in myeloid and platelets cells in the peripheral blood and myeloid hyperplasia in the bone marrow $[1,2]$.

The pathophysiology of CML has been well established since the description of the Philadelphia chromosome (or $\mathrm{Ph}^{1}$ ) by Nowell and Hungerford in 1960 [3]. The disease is characterized by a translocation [4] that consists of a juxtaposition of the $A B L 1$ gene from chromosome 9 and the $B C R$ gene from chromosome 22, coding for a protein with constitutive tyrosine kinase activity, able to be targeted by tyrosine kinase inhibitors $[5,6]$.

Depending on the site of the breakpoint in the $B C R$ gene, the fusion protein can vary in size from $185 \mathrm{kDa}$ to $230 \mathrm{kDa}$. The break most commonly occurs between exon 13 (e13) and exon 14 (e14 formerly known as b2) or between e14 and exon 15 (e14, formerly known as b3) in a region of approximately $5.8 \mathrm{~kb}$ called the major breakpoint cluster or M-BCR. The breakpoint in the $A B L 1$ gene is generally located between exons a1 and a2.

Therefore, most of the patients with chronic phase (CP)-CML express a 210-kDa BCR-ABL1 (p210 ${ }^{\text {BCR-ABL1) }}$ coded by e13a 2 or e $14 \mathrm{a} 2 B C R-A B L 1$ transcripts. In some cases, both transcripts can be co-expressed [7,8]. These two different transcripts differ by a 25 -extra aminoacids insertion coded by the e14 (b3) exon. A recent study conducted in Europe on more than 45000 CML cases showed a proportion of $39 \%$ of e $13 \mathrm{a} 2,62 \%$ of e $14 \mathrm{a} 2$ and e14a2/e13a2 transcript, and this distribution differed by gender and by age [8].

The prognostic impact of the type of $B C R-A B L 1$ transcript has been the subject of controversies over time. However, in the imatinib era, several studies on a limited number of patients have suggested a deeper and faster response in patients expressing e14a2 [9-14].

Nilotinib (Tasigna ${ }^{\circledR}$; Novartis, East Hanover, NJ, USA) is a BCR-ABL1 inhibitor designed to be more potent and selective than imatinib [15] and shows better efficacy than imatinib for the treatment of patients with newly diagnosed CML in chronic phase [16]. To our knowledge, there are few published data analyzing the influence of transcripts type in patients treated by nilotinib frontline.

The aim of this study was to evaluate the prognostic impact of transcripts type e $14 \mathrm{a} 2$ or e $13 \mathrm{a} 2$ in patients with chronic phase CP-CML treated frontline with nilotinib.

\section{RESULTS}

\section{PCR efficiency}

As shown in Figure 1, no differences in terms of PCR efficiencies were observed between the two transcripts, avoiding a bias of amplification that could explain the difference in transcript quantification $(p=$ .32). Furthermore, PCR efficiencies were the not different $(p=.34)$ when we used a specific e14a2 primer (slope $=$ $-3,49$; efficiency $93 \%$ ) or the EAC primers (slope $=-3,46$; efficiency $=94 \%$ ) on a e14a 2 cell line, suggesting that amplification is comparable between the two transcripts with EAC protocol.

\section{Patients and disease characteristics}

Overall, 118 patients treated frontline with nilotinib for CP-CML were included in this study. Four patients were excluded from this analysis: 3 were treated by interferon $(n=2)$ or by cytarabine $(n=1)$ and 1 patient was in accelerated phase. All analyses were made on the remaining 114 patients. The baseline characteristics of the patients are summarized in Table 1.

Sixty-three patients $(55.3 \%)$ expressed e14a2, and 51 patients $(44.7 \%)$ expressed e13a2 transcripts. The median age at diagnosis was 51 years (range: $18-84$ years) for the entire cohort and $52.6 \%$ of the patients were male with no significant differences $(p=.27$ and $p=.95$, respectively).

At the time of diagnosis, patients expressing e14a2 had a median white blood cell (WBC) count of $106 \mathrm{G} / \mathrm{L}$ compared to $156 \mathrm{G} / \mathrm{L}$ in the e13a2 group. Median platelet count was $405 \mathrm{G} / \mathrm{L}$ in the e $14 \mathrm{a} 2$ group and $311 \mathrm{G} / \mathrm{L}$ in the e13a2 group. These differences were not significant $(p=$ $.17, p=.14$, respectively) between the 2 subgroups.

The patient distribution according to ELTS [17], EUTOS [18], Hasford [19] and Sokal [20] scores was comparable between groups.

There was a similar rate of additional clonal abnormality (ACA) and variant translocation ( $p=.35$ ).

Median follow-up was 49 months for the entire cohort (range: 3-121 months). The median duration of treatment with nilotinib was 42 months (range: 3-111 months) for e $14 \mathrm{a} 2$ patients and 49 months (range: $2-110$ months) for e13a2 patients $(p=.47)$. At diagnosis, all patients received $600 \mathrm{mg}$ of nilotinib per day $(2 \times 300$ $\mathrm{mg}$ ), and during follow up 6 patients (4 in e13a2 subgroup and 2 in e14a2) needed a dose reduction to $450 \mathrm{mg} / \mathrm{day}$ for drug toxicity.

\section{Response to treatment}

According to the absence of a systematic difference in prognostic scores, the transcript groups were compared without stratification for those covariates. Only patients with data available at the time of assessment were included.

Response rates for endpoints considered as optimal by the ELN are summarized in Table 2. CHR and CCyR rates were similar in both groups $(p=.57$ and $p=.52$, respectively). There were no differences when considering 
the EMR between groups, with rates as follows: e14a2 $(85.5 \%)$ and e13a2 (88\%) $(p=.46)$.

Patients expressing e13a 2 had a significantly lower rate of MMR at 12 months $(50.1 \%)$ compared to those expressing e14a2 $(66.7 \%)(p=.048)$.

We then analyzed the cumulative incidence of response according to the type of transcript (Figure 2). The cumulative incidence of MMR was $100 \%$ in the e14a2 group compared to $84.1 \%$ for the e $13 \mathrm{a} 2$ group ( $p=$ .007 ) with a median time of 6.7 months and 17.1 months, respectively.

The cumulative incidence of $\mathrm{MR}^{4.5}$ was also higher in the e14a2 group (100\%) than in the e13a2 group $(59.9 \%)(p=.005)$ with a median time to $\mathrm{MR}^{4.5}$ of 39.7 months and 70.9 months, respectively.

One patient was lost during follow-up and 2 patients progressed: accelerated phase $(n=1)$ and a transformation in $\mathrm{Ph}^{+} \mathrm{ALL}(n=1)$. These patients underwent allogenic stem cell transplantation. Two patients $(1.6 \%)$ died during the follow-up: one from a transformation in $\mathrm{Ph}^{+}$acute lymphoid leukemia (ALL) and one from uterine cancer.

The estimated 5-year overall survival (OS) was $95.6 \%$ for the e14a2 group and $100 \%$ for the e13a2 group (Figure 3A). The 5-year event-free survival for the e14a2 group was $72.5 \%$ and $66.8 \%$ for the e13a2 group (Figure 3B). We did not observed differences in OS $(p=.20)$ or EFS $(p=.84)$ according to transcript type in our cohort.

\section{TKI discontinuation}

Thirty-nine patients (34.2\%) discontinued nilotinib during the follow-up and there was no difference between groups $(p=.19)$ with 23 patients over $63(36.5 \%)$ in the e $14 \mathrm{a} 2$ group and 16 patients over $51(31.4 \%)$ in the e13a2 group. The reasons to stop therapy were failure for $28.2 \%(n=11)$, treatment side effects for $35.9 \%$ ( $n$ $=14$ ), an attempt of treatment free remission (TFR) due to sustained deep molecular response for $33.3 \%(n=13)$ and pregnancy for $2.6 \%(n=1)$. Results are summarized in Table 3.

\section{MMR and $M^{4.5}$ : univariate and multivariate analysis}

We then performed univariate and multivariate analysis to examine the role of the transcript's type on achieving molecular responses.

In the multivariate model, a high platelet count of over $300 \mathrm{G} / \mathrm{L}$ was the only factor significantly predicting better MMR rates (Supplementary Table 1). Considering $\mathrm{MR}^{4.5}$, our multivariate model showed that expressing

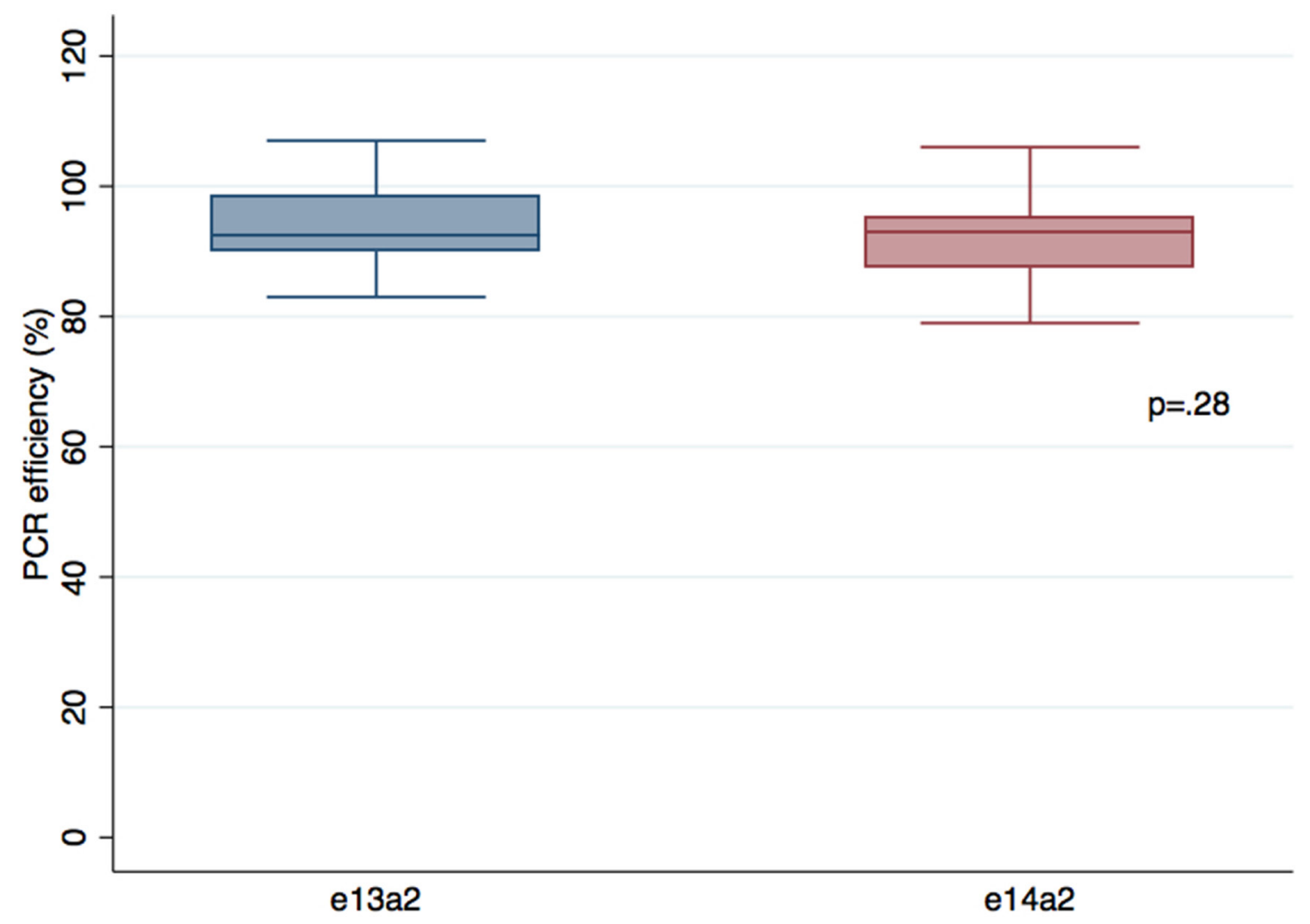

Figure 1: Comparison of PCR efficiencies between e13a2 and e14a2 transcripts. Serial 10 fold dilutions of two different cell lines expressing e13a2 or e14a2 transcripts were performed in 4 distinct experiments $(n=48)$. Results were compared using the WilcoxonMann-Whitney test. 
Table 1: Clinical and Biological Characteristics of patients at diagnosis according to transcript subtype (e13a2 versus e14a2) $(n=114)$

\begin{tabular}{|c|c|c|c|}
\hline \multirow[b]{2}{*}{ Characteristic } & \multicolumn{3}{|c|}{$N=(\%)$ or median [range] } \\
\hline & e14a2 & e13a2 & $p=$ \\
\hline$N$ & $63(55.3)$ & $51(44.7)$ & \\
\hline Gender, male & $33(52.4)$ & $27(52.9)$ & .95 \\
\hline Age (years) & 49 [19-84] & $53[18-78]$ & .27 \\
\hline Hemoglobin $(g / L)$ & 118 [69-157] & 114 [79-156] & .48 \\
\hline WBC count $\left(10^{3} / \mu \mathrm{L}\right)$ & $106[4.5-623]$ & $156[17.6-552.5]$ & .17 \\
\hline PLT count $\left(10^{3} / \mu \mathrm{L}\right)$ & 405 [83-1999] & 311 [104-1315] & .14 \\
\hline Peripheral blasts (\%) & $1.0[0-13]$ & $1.1[0-7]$ & .98 \\
\hline \multicolumn{4}{|l|}{ ELTS score } \\
\hline Low & $32(52.5)$ & $24(48)$ & .87 \\
\hline Intermediate & $21(34.4)$ & $18(36)$ & \\
\hline High & $8(13.1)$ & $8(16)$ & \\
\hline \multicolumn{4}{|l|}{ EUTOS score } \\
\hline Low & $55(90.2)$ & $45(90)$ & .98 \\
\hline High & $6(9.8)$ & $5(10)$ & \\
\hline \multicolumn{4}{|l|}{ Sokal score } \\
\hline Low & $21(34.4)$ & $16(32)$ & .84 \\
\hline Intermediate & $27(44.3)$ & $21(42)$ & \\
\hline High & $13(21.3)$ & $13(26)$ & \\
\hline \multicolumn{4}{|l|}{ Hasford score } \\
\hline Low & $24(39.3)$ & $13(26)$ & .08 \\
\hline Intermediate & $25(41)$ & $31(62)$ & \\
\hline High & $12(19.7)$ & $6(12)$ & \\
\hline $\begin{array}{l}\text { ACA Ph+ } \\
\text { or variant translocation }\end{array}$ & $8(13.1)$ & $10(20)$ & .35 \\
\hline Median duration of treatment (months) & $42[3-111]$ & $49[2-104]$ & .47 \\
\hline Median follow-up (months) & $46[3-121]$ & $53[3-111]$ & .97 \\
\hline
\end{tabular}

Abbreviations: ACA Ph+, additional chromosome abnormalities in $\mathrm{Ph}+$ cells; EUTOS, European Treatment and Outcome Study; ELTS, EUTOS long-term survival; PLT, platelets; WBC, white blood cells.

e14a2 transcript was predictive of $\mathrm{MR}^{4.5}$ among other variables: sex (male), high WBC count and high platelet count. These results are summarized in Table 4.

\section{DISCUSSION}

A majority of patients with CP-CML expressed e14a2 or e13a2 $B C R-A B L 1$ transcript. The influence of a transcript type on outcome or molecular response has been questioned for a long time. In the Imatinib era, numerous studies suggested better and faster responses in patients expressing e14a2 transcript as discussed in a recent review by Ercaliskan et al. [21].

As few data evaluating the impact of transcript types in patients treated by second tyrosine kinase inhibitors were available, we retrospectively evaluated in this study the influence of transcript type in 114 patients with CP-CML treated frontline with Nilotinib. Patients expressing e14a2 transcripts had deeper and faster molecular responses, when considering MMR and $\mathrm{MR}^{4.5}$, compared to those expressing e13a2, despite the use of second-generation TKI. These results are consistent with data reported by Jain et al. [22]. Similarly, out of 237 patients, Yi-Jiun Su et al. [23] found that e14a2 patients had a better rate of MMR at 12 months compared to e13a2 patients despite no difference in median time to MMR, median time to $\mathrm{MR}^{4.5}$ or in the cumulative incidence of $\mathrm{MR}^{4.5}$. A preliminary study from the GIMENA CML WP [24] suggested that patients expressing e13a2 had a trend to a lower cumulative incidence of MMR and $\mathrm{MR}^{4.0}$ but these differences were not significant. However, these results became significant when grouping together 
Table 2: Hematological, Cytogenetic and molecular responses at 3, 6, and 12 months follow-up for patients according to transcript type (e13a2 versus e14a2) and ELN endpoints

\begin{tabular}{cccccccc}
\hline & \multicolumn{2}{c}{ CHR at 3 months } & \multicolumn{2}{c}{ CCyR at 6 months } & \multicolumn{2}{c}{ MMR at 12 months } \\
\cline { 2 - 8 } & $\mathbf{e 1 4 a 2}$ & $\mathbf{e 1 3 a 2}$ & $\mathbf{e 1 4 a 2}$ & $\mathbf{e 1 3 a 2}$ & $\mathbf{e 1 4 a 2}$ & $\mathbf{e 1 3 a 2}$ \\
\hline$N$ & $58 / 62$ & $48 / 50$ & $54 / 58$ & $43 / 48$ & $42 / 63$ & $26 / 51$ \\
$\%$ & 93.5 & 96 & 93.1 & 89.6 & 66.7 & 50.1 \\
$p=$ & & .57 & & & .52 & & .048 \\
\hline
\end{tabular}

Abbreviations: CHR, complete hematological response; CCyR, complete cytogenetic response; MMR, major molecular response. Results were compared using Student test.

patients expressing e $14 \mathrm{a} 2$ and patient co-expressing both transcripts.

One explanation to our findings could be linked to the differential amplification between the two transcripts. As suggested by Hanfstein et al. [10], we speculated that a better amplification of the smaller transcript (i.e. e13a2) could result in a majored and biased quantification. Indeed, the European Against Cancer (EAC) protocol used a common forward primer in $B C R$ exon 13 , resulting in a 76 bp difference in amplification between the 2 transcripts. Therefore, we first evaluated PCR amplification efficiencies and found no significant difference between e14a 2 and e13a2 transcripts, allowing the direct comparison of quantification values whatever the transcript type.

$\mathbf{A}$

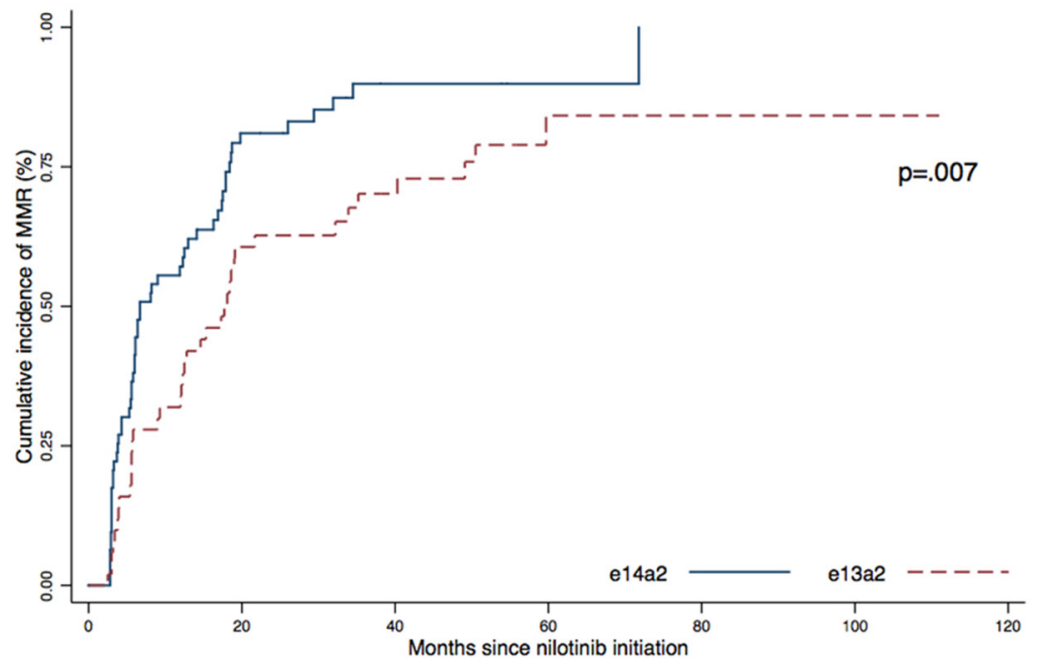

B

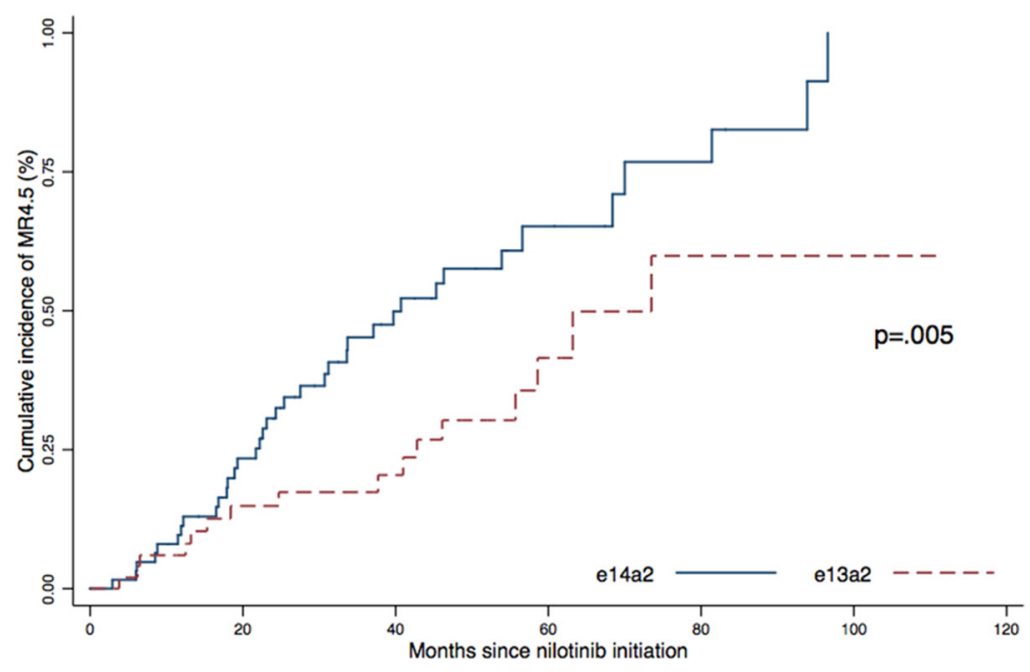

Figure 2: Cumulative incidence of MMR (A) and MR4.5 (B) according to transcript type (e13a2 or e14a2) were obtained using the Kaplan-Meier method, results were compared using the log-Rank test. 
When considering the cumulative probability of achieving $\mathrm{MR}^{4.5}$, it is interesting to see that the clearance profile of $B C R-A B L 1$ is very similar in the first 18 months between the two transcript subtypes. This exponential biphasic declination pattern had already been identified with imatinib [25] and confirmed independently of the TKI given frontline [26]. The authors explained this phenomenon by the elimination of differentiated leukemia cells first and then the leukemia progenitors.

Some data are attractive trails to explain the differential clearance of both types of transcripts. Interestingly, Lucas et al. [12] evaluated the pCrKL/CrKL ratio, a surrogate of BCR-ABL1 kinase activity [27], and found that patients expressing e $13 \mathrm{a} 2$ had a higher tyrosine kinase activity. The spatial structure of the 2 proteins seems to be different, as illustrated by Hai et al. [28], and could impact the interaction with kinase inhibitors.
More recently, Pagani et al. [29] described, even in a small number of patients, a lower $B C R-A B L 1 \mathrm{mRNA} /$ $B C R-A B L 1$ DNA ratio in e13a 2 versus e $14 \mathrm{a} 2$ patients, consistent with an experimental low-BCR-ABL1 murine model showing a reduced imatinib sensitivity. This low $B C R-A B L 1$ expression in e13a2 patients could lead to incorrect classification for treatment decisions, including $\mathrm{MR}^{4.5}$ or $\mathrm{MR}^{5}$ evaluations that guide TKI discontinuation.

The role of the additional 25 amino-acids (coded by the e14 exons) between e14a2 and e13a2 transcripts has also been questioned. Clark et al. first demonstrated a specific immunologic role of e14a 2 peptide compared to e $13 \mathrm{a} 2$ and its potential to induce cytotoxic T-lymphocyte response [30]. This Immunologic role could also have an impact on TFR.

Our study includes several limitations. Molecular evaluation was not performed centrally. However, the techniques used are similar and there is a national and

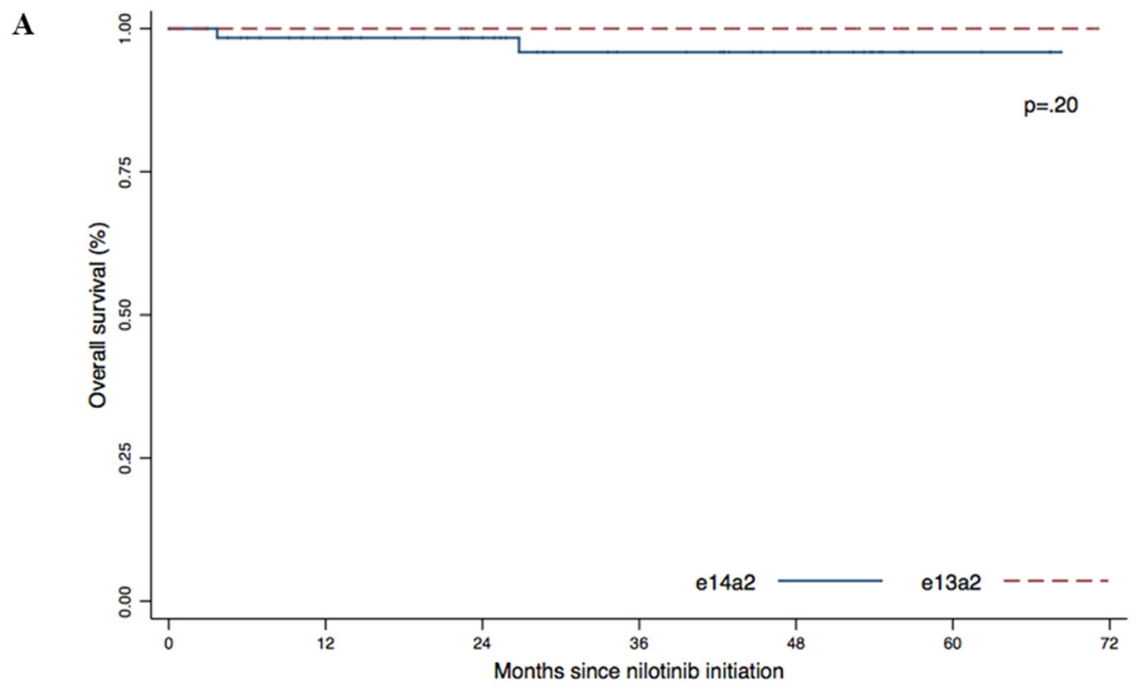

B

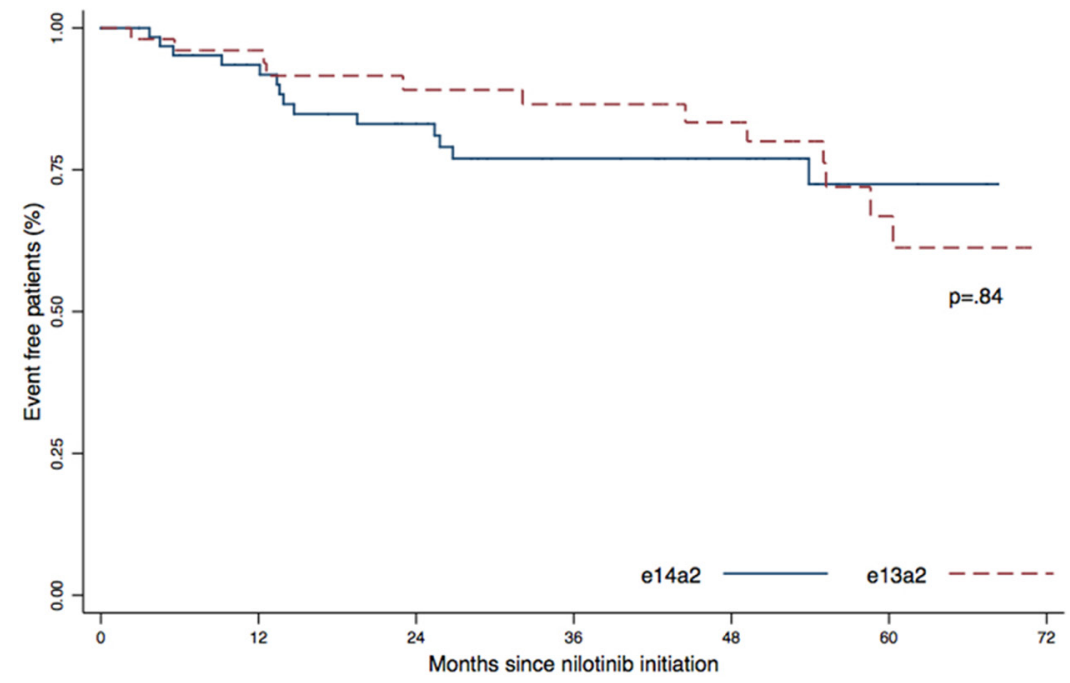

Figure 3: Overall survival (A) and Event Free Survival (B) according to transcript type (e13a2 versus e14a2). $P$-values were obtained using the log-Rank test. 


\begin{tabular}{lccc}
\hline Causes of TKI discontinuation & $\mathbf{e 1 4 a 2}$ & $\boldsymbol{N}=\mathbf{( \% )}$ & $\boldsymbol{p}=$ \\
\hline Failure & $7(30.4)$ & $\mathbf{e 1 3 \mathbf { 2 }}$ & \\
Side-effect & $6(26.1)$ & $8(25)$ & \\
TFR-attempt & $10(43.5)$ & $3(18.8)$ & \\
Other & $0(0)$ & $1(6.2)$ & .19 \\
Total & $23(100)$ & $16(100)$ & \\
\hline
\end{tabular}

Abbreviations: TFR, treatment-free remission; TKI, tyrosine kinase inhibitor. Results were compared using Fischer's exact method.

international consensus on their implementation [31]; all the results are aligned on the international scale by the 5 laboratories involved in our study. Given the effectiveness of current treatments and especially with second-generation inhibitors, it is necessary to have a sufficient number of patients and in this context, our retrospective cohort on a limited number of patients have to be confirmed in a larger one, as what had been done with imatinib.

We only focused on nilotinib, the only second generation TKI approved and reimbursed in France. It may be interesting to evaluate the impact of other drugs approved as first line therapies by the FDA or the EMA such as dasatinib or bosutinib. Finally, the mechanism underlying the different response rates to treatment among molecular subgroups need to be better understood.

Overall, we conclude that even treated with nilotinib first line, patients with CP-CML expressing $B C R-A B L 1$ e13a2 transcript seems to have a lower rate of MMR and $\mathrm{MR}^{4.5}$.

\section{MATERIALS AND METHODS}

\section{Patients}

This is a retrospective and multicentric study. All patients with newly diagnosed CP-CML and treated frontline with nilotinib in Annecy, Clermont-Ferrand, Lyon, Saint-Etienne and Toulouse hospitals were enrolled. The patients were diagnosed between 2007 and 2017 and treated inside or outside clinical trials. Patients expressing e14a2 and e13a2 transcripts were analyzed. Patients with atypical $B C R-A B L 1$ and co-expressors transcripts or treated in combination with interferon were excluded from this analysis. Demographic and disease characteristics were assessed at baseline. Written information has been given to all participants and a non-opposition statement was obtained according to national and institutional requirements.

\section{Response criteria}

Response criteria were based on the 2013 European Leukemia Net (ELN) recommendations for the management of CML [32]. Complete hematological response (CHR) was defined by platelets $<450 \mathrm{G} / \mathrm{L}$, white cells $<10 \mathrm{G} / \mathrm{L}$, no circulating immature myeloid cells, $<5 \%$ basophils on differential and no palpable splenomegaly. Complete cytogenetic response (CCyR) was defined by no $\mathrm{Ph}^{1}$ cells in karyotype. Early molecular response (EMR) was characterized by a $B C R-A B L 1^{\text {IS }} \leq$ $10 \%$ at 3 months. Major molecular response (MMR) was obtained when $B C R-A B L 1^{\text {IS }}$ was lower or equal to $0.10 \%$ and $\mathrm{MR}^{4.5}$ with a BCR-ABL $1^{\text {IS }}<0.0032 \%$.

All molecular classifications were based on $B C R$ $A B L 1$ control ratios and standardized according to the International Scale or IS [33]. Follow-up was similar in all five centers with at least real-time quantitative PCR performed at 3, 6, 12 and 18 months and cytogenetic analysis at 6 months and until CCyR. Event free survival (EFS) was measured from the initiation of treatment to the date of any of the following events while on therapy: loss of CHR, loss of CCyR, resistance, progression to accelerated or blastic phase, stopping nilotinib for toxicity or death from any cause. Overall survival (OS) was defined from the time of treatment initiation to the date of death from any cause at any time or date of last follow-up.

\section{Transcripts typing}

RNA extraction from Ficoll-separated peripheral blood cells and reverse transcription were performed at the time of diagnosis for the majority of patients or retrospectively from a sample collected at the diagnosis when the results were not available. PCR were performed using Veriti thermal cycler (Applied Biosystems, Carlsbad, CA, USA).

The type of $B C R-A B L 1$ transcript was identified according to the size of RT-PCR product as previously described [34].

\section{Transcripts quantification}

Both Transcripts were quantified in blood samples using EAC protocol [35] MMLV-RT was changed for a Superscript VILO-RT (Life TechnologiesThermo Fischer Sicentific) to ensure a minimum of 32000 copies of $A B L 1$ control gene to define at least $\mathrm{MR}^{4.5}$ for each sample. All results are expressed and standardized according to IS. 
Table 4: Multivariate analysis to identify factors predictive of $\mathrm{MR}^{4.5}$

\begin{tabular}{|c|c|c|c|c|c|}
\hline Univariate analysis & OR & {$[95 \%$ CI OR] } & $\mathrm{MR}^{4.5} \mathrm{~N}(\%)$ & No $\mathrm{MR}^{4.5} \mathrm{~N}(\%)$ & $p=$ \\
\hline \multicolumn{6}{|l|}{ Male } \\
\hline Yes & 0.19 & {$[0.09-0.41]$} & $20(33.3)$ & $47(72.3)$ & $<.001$ \\
\hline No & & & $40(66.7)$ & $18(27.7)$ & \\
\hline \multicolumn{6}{|l|}{ Splenomegaly } \\
\hline Yes & 0.29 & {$[0.11-0.79]$} & $6(10)$ & $18(27.7)$ & .02 \\
\hline No & & & $54(90)$ & $47(72.3)$ & \\
\hline \multicolumn{6}{|l|}{$\begin{array}{l}\text { WBC count }(>150 \times \\
\left.10^{9} / \mathrm{L}\right)\end{array}$} \\
\hline Yes & 0.22 & {$[0.10-0.49]$} & $13(21.7)$ & $36(55.4)$ & $<.001$ \\
\hline No & & & $47(78.3)$ & $29(44.6)$ & \\
\hline \multicolumn{6}{|l|}{ PLT count $(>300 \mathrm{G} / \mathrm{L})$} \\
\hline Yes & 5.20 & {$[2.29-11.75]$} & $49(81.7)$ & $30(46.2)$ & $<.001$ \\
\hline No & & & $11(18.3)$ & $35(53.8)$ & \\
\hline \multicolumn{6}{|c|}{ Hemoglobin $(>120 \mathrm{~g} / \mathrm{L})$} \\
\hline Yes & 1.50 & {$[0.73-3.05]$} & $29(48.3)$ & $25(38.5)$ & .27 \\
\hline No & & & $31(51.7)$ & $40(61.5)$ & \\
\hline \multicolumn{6}{|l|}{ High ELTS score } \\
\hline Yes & 0.40 & {$[0.13-1.21]$} & $5(8.3)$ & $12(18.5)$ & .11 \\
\hline No & & & $55(91.7)$ & $53(81.5)$ & \\
\hline \multicolumn{6}{|l|}{ High Sokal score } \\
\hline Yes & 0.71 & {$[0.30-2.00]$} & $12(20)$ & $17(26.1)$ & .42 \\
\hline No & & & $48(80)$ & $48(73.9)$ & \\
\hline \multicolumn{6}{|l|}{$\begin{array}{l}\text { ACA or variant } \\
\text { translocation }\end{array}$} \\
\hline Yes & 0.73 & {$[0.27-1.95]$} & $8(13.3)$ & $11(17.5)$ & .53 \\
\hline No & & & $52(86.7)$ & $52(82.5)$ & \\
\hline \multicolumn{6}{|c|}{$\begin{array}{l}\text { Transcript e14a2, alone } \\
\text { or co-expressed }\end{array}$} \\
\hline Yes & 3.21 & {$[1.51-6.80]$} & $44(73.3)$ & $30(46.2)$ & .002 \\
\hline No & & & $16(26.7)$ & $35(53.8)$ & \\
\hline Multivariate analysis & OR & & [95\% CI OR] & & $p=$ \\
\hline Male & 0.28 & & {$[0.11-0.71]$} & & .007 \\
\hline Splenomegaly & 0.56 & & {$[0.13-2.46]$} & & .45 \\
\hline WBC count & 0.29 & & {$[0.10-0.84]$} & & .02 \\
\hline Platelets & 3.68 & & [1.39-9.76] & & .009 \\
\hline ELTS score & 2.42 & & [0.43-13.38] & & .31 \\
\hline e14a2 & 3.25 & & {$[1.30-8.18]$} & & .01 \\
\hline
\end{tabular}

Abbreviations: ACA, additional chromosome abnormalities in $\mathrm{Ph}+$ cells; EUTOS, European Treatment and Outcome Study; ELTS; PLT, platelets; WBC, white blood cells. Variables with $p$ values inferior to 0.20 in univariate analysis were included in the multivariate model.

In order to investigate a possible bias in amplification according to transcript type, RQ-PCR efficiencies for e13a2 and e14a2 were compared, based on the slope of the standard curves resulting from serial dilutions of
cDNA extracted from K562 (e14a2) and BV173 (e13a2) cell lines. A total of 48 standard curves ( 24 for e13a2 and 24 for e14a2) in 4 different experiments were performed. Furthermore, to demonstrate the ability of the EAC assay 
to accurately determine the number of longer transcripts produced by an e14a2 fusion RNA, we designed a specific assay for e14a2 transcript (Forward Primer: ATGGGTTTCTGAATGTCATCG) and compared the PCR efficiencies between EAC protocol and specific e14a2 protocol in a e14a 2 cell line (K562). A total of 10 standard curves in two different experiments were performed.

\section{Statistical analysis}

PCR efficiencies were compared using the Wilcoxon-Mann-Whitney test. Baseline characteristics of patients according to the type of transcripts were performed using Pearson's chi square test, Kruskal-Wallis test or one way-ANOVA, as appropriate.

The cumulative incidence of MMR, MR ${ }^{4.5}$, EFS and OS was estimated using the Kaplan-Meier method. Patients who discontinued treatment whatever the cause were censored at the date of discontinuation. Differences were compared with the log-rank test.

Univariate and multivariate analyses were performed to identify whether the subtype of transcript could predict the molecular responses. The unadjusted significance level of 0.05 was applied to all statistical tests.

Statistical analyses were carried out using STATA/ SE version 14.1 (Stata corp., College Station, TX, USA).

\section{Abbreviations}

ACA: Additional Clonal Abnormality; ALL: Acute Lymphoblastic Leukemia; CCyR: Complete Cytogenetic Response; CHR: Complete Hematologic Response; DNA: DeoxyriboNucleic acid; ELN: European leukemia Net; ELTS: Eutos Long Terme Survival; EUTOS: EUropean Treatment Outcome Study; MR: Molecular response; MMR: Major molecular response; PCR: polymerase Chain reaction; RNA: RiboNucleic acid; RT-PCR: Reverse Transcriptase Polymerase Chain reaction; TKI: Tyrosine Kinase Inhibitors; TFR: Treatment free remission; WBC: White Blood Count.

\section{Author contributions}

Drs. PF, DG and AG had full access to all the data in the study and take responsibility for the integrity of the data and the accuracy of the data analysis. DG and PF contributed to the study concept and design. DG, PF, $\mathrm{AG}$ and FN contributed to the acquisition, analysis, or interpretation of data. AG, PF and DG contributed to the drafting of the manuscript. AG and DG contributed to the statistical analysis. All authors read, participated in the revision and approved the final manuscript.

\section{ACKNOWLEDGMENTS}

We acknowledge Mr. Armand Glasson and the association Anim'Montbernier (Ruy Montceau, France) for its partial support for this study.

\section{CONFLICTS OF INTEREST}

Authors have no conflicts of interest to declare.

\section{REFERENCES}

1. Sawyers CL. Chronic Myeloid Leukemia. N Engl J Med. 1999; 340:1330-1340. https://doi.org/10.1056/ NEJM199904293401706. [PubMed]

2. Hehlmann R, Hochhaus A, Baccarani M, and European LeukemiaNet. Chronic myeloid leukaemia. Lancet. 2007; 370:342-50. https://doi.org/10.1016/S0140-6736(07)61165-9. [PubMed]

3. Nowell PC. Discovery of the Philadelphia chromosome: a personal perspective. J Clin Invest. 2007; 117:2033-2035. https://doi.org/10.1172/JCI31771. [PubMed]

4. Rowley JD. Letter: A new consistent chromosomal abnormality in chronic myelogenous leukaemia identified by quinacrine fluorescence and Giemsa staining. Nature. 1973; 243:290-293. https://doi.org/10.1038/243290a0. [PubMed]

5. Daley GQ, Van Etten RA, Baltimore D. Induction of chronic myelogenous leukemia in mice by the $\mathrm{P} 210 \mathrm{bcr} / \mathrm{abl}$ gene of the Philadelphia chromosome. Science. 1990; 247:824-830. https://doi.org/10.1126/science.2406902. [PubMed]

6. Heisterkamp N, Groffen J, Stephenson JR, Spurr NK, Goodfellow PN, Solomon E, Carritt B, Bodmer WF. Chromosomal localization of human cellular homologues of two viral oncogenes. Nature. 1982; 299:747-749. https:// doi.org/10.1038/299747a0. [PubMed]

7. Deininger MW, Goldman JM, Melo JV. The molecular biology of chronic myeloid leukemia. Blood. 2000; 96:3343-3356. https://doi.org/10.1182/blood.V96.10.3343. [PubMed]

8. Baccarani M, Castagnetti F, Gugliotta G, Rosti G, Soverini S, Albeer A, Pfirrmann M; International BCR-ABL Study Group. The proportion of different BCR-ABL1 transcript types in chronic myeloid leukemia. An international overview. Leukemia. 2019; 33:1173-1183. https://doi. org/10.1038/s41375-018-0341-4. [PubMed]

9. Melo JV. BCR-ABL gene variants. Baillieres Clin Haematol. 1997; 10:203-222. https://doi.org/10.1016/ $\underline{\text { S0950-3536(97)80003-0. }}$

10. Hanfstein B, Lauseker M, Hehlmann R, Saussele S, Erben P, Dietz C, Fabarius A, Proetel U, Schnittger S, Haferlach C, Krause SW, Schubert J, Einsele H, et al, and SAKK and the German CML Study Group. Distinct characteristics of e13a2 versus e14a2 BCR-ABL1 driven chronic myeloid leukemia under first-line therapy with imatinib. Haematologica. 2014; 99:1441-1447. https://doi. org/10.3324/haematol.2013.096537. [PubMed]

11. Castagnetti F, Gugliotta G, Breccia M, Iurlo A, Levato L, Albano F, Vigneri P, Abruzzese E, Rossi G, Rupoli S, Cavazzini F, Martino B, Orlandi E, et al, and GIMEMA CML Working Party. The BCR-ABL1 transcript type 
influences response and outcome in Philadelphia chromosome-positive chronic myeloid leukemia patients treated frontline with imatinib. Am J Hematol. 2017; 92:797-805. https://doi.org/10.1002/ajh.24774. [PubMed]

12. Lucas CM, Harris RJ, Giannoudis A, Davies A, Knight K, Watmough SJ, Wang L, Clark RE. Chronic myeloid leukemia patients with the e13a2 BCR-ABL fusion transcript have inferior responses to imatinib compared to patients with the e14a2 transcript. Haematologica. 2009; 94:1362-1367. https://doi.org/10.3324/haematol.2009.009134. [PubMed]

13. Mir R, Ahmad I, Javid J, Zuberi M, Yadav P, Shazia R, Masroor M, Guru S, Ray PC, Gupta N, Saxena A. Simple multiplex RT-PCR for identifying common fusion BCRABL transcript types and evaluation of molecular response of the $\mathrm{a} 2 \mathrm{~b} 2$ and $\mathrm{a} 2 \mathrm{~b} 3$ transcripts to Imatinib resistance in north Indian chronic myeloid leukemia patients. Indian J Cancer. 2015; 52:314-318. [PubMed]

14. Pagnano KBB, Miranda EC, Delamain MT, Duarte GO, de Paula EV, Lorand-Metze I, de Souza CA. Influence of BCRABL Transcript Type on Outcome in Patients With ChronicPhase Chronic Myeloid Leukemia Treated With Imatinib. Clin Lymphoma Myeloma Leuk. 2017; 17:728-733. https:// doi.org/10.1016/i.clml.2017.06.009. [PubMed]

15. Manley PW, Stiefl N, Cowan-Jacob SW, Kaufman S, Mestan J, Wartmann M, Wiesmann M, Woodman R, Gallagher N. Structural resemblances and comparisons of the relative pharmacological properties of imatinib and nilotinib. Bioorg Med Chem. 2010; 18:6977-6986. https:// doi.org/10.1016/i.bmc.2010.08.026. [PubMed]

16. Kantarjian HM, Hochhaus A, Saglio G, De Souza C, Flinn IW, Stenke L, Goh YT, Rosti G, Nakamae H, Gallagher NJ, Hoenekopp A, Blakesley RE, Larson RA, et al. Nilotinib versus imatinib for the treatment of patients with newly diagnosed chronic phase, Philadelphia chromosomepositive, chronic myeloid leukaemia: 24-month minimum follow-up of the phase 3 randomised ENESTnd trial. Lancet Oncol. 2011; 12:841-851. https://doi.org/10.1016/S14702045(11)70201-7. [PubMed]

17. Pfirrmann M, Baccarani M, Saussele S, Guilhot J, Cervantes F, Ossenkoppele G, Hoffmann VS, Castagnetti F, Hasford J, Hehlmann R, Simonsson B. Prognosis of long-term survival considering disease-specific death in patients with chronic myeloid leukemia. Leukemia. 2016; 30:48-56. https://doi. org/10.1038/leu.2015.261. [PubMed]

18. Hasford J, Baccarani M, Hoffmann V, Guilhot J, Saussele S, Rosti G, Guilhot F, Porkka K, Ossenkoppele G, Lindoerfer D, Simonsson B, Pfirrmann M, Hehlmann R. Predicting complete cytogenetic response and subsequent progressionfree survival in 2060 patients with CML on imatinib treatment: the EUTOS score. Blood. 2011; 118:686-692. https://doi.org/10.1182/blood-2010-12-319038. [PubMed]

19. Hasford J, Pfirrmann M, Hehlmann R, Allan NC, Baccarani M, Kluin-Nelemans JC, Alimena G, Steegmann JL, Ansari $\mathrm{H}$, and Writing Committee for the Collaborative CML Prognostic Factors Project Group. A new prognostic score for survival of patients with chronic myeloid leukemia treated with interferon alfa. J Natl Cancer Inst. 1998; 90:850-58. https://doi.org/10.1093/jnci/90.11.850. [PubMed]

20. Sokal JE, Cox EB, Baccarani M, Tura S, Gomez GA, Robertson JE, Tso CY, Braun TJ, Clarkson BD, Cervantes F. Prognostic discrimination in "good-risk" chronic granulocytic leukemia. Blood. 1984; 63:789-799. https:// doi.org/10.1182/blood.V63.4.789.789. [PubMed]

21. Ercaliskan A, Eskazan AE. The impact of BCR-ABL1 transcript type on tyrosine kinase inhibitor responses and outcomes in patients with chronic myeloid leukemia. Cancer. 2018; 124:3806-3818. https://doi.org/10.1002/ cncr.31408. [PubMed]

22. Jain P, Kantarjian H, Patel KP, Gonzalez GN, Luthra R, Shamanna RK, Sasaki K, Jabbour E, Romo CG, Kadia TM, Pemmaraju N, Daver N, Borthakur G, et al. Impact of BCR-ABL transcript type on outcome in patients with chronic-phase CML treated with tyrosine kinase inhibitors. Blood. 2016; 127:1269-1275. https://doi.org/10.1182/ blood-2015-10-674242. [PubMed]

23. Su YJ, Kuo MC, Teng CL, Chen TY, Ma MC, Hsiao PC, Wang MC, Lin TH, Hwang WL, Chen CC, Yang Y, Pei SN, Huang YM, et al. Comparison of Molecular Responses between e14a2 and e13a2 BCR-ABL1 Transcripts in Patients with Chronic Myeloid Leukemia in Chronic Phase Treated with Front-Line Second Generation Tyrosine Kinase Inhibitors: Taiwan CML Study. Blood. 2017; 130:1603-1603.

24. Castagnetti F, Gugliotta G, Breccia M, Stagno F, D'Adda M, Levato L, Carella AM, Martino B, Tiribelli M, Fava C, Binotto G, Avanzini P, Bocchia M, et al. Prognostic Value of BCRABL1 Transcript Type in Chronic Myeloid Leukemia Patients Treated Frontline with Nilotinib. Blood. 2016; 128:3070 3070. https://doi.org/10.1182/blood.V128.22.3070.3070.

25. Michor F, Hughes TP, Iwasa Y, Branford S, Shah NP, Sawyers CL, Nowak MA. Dynamics of chronic myeloid leukaemia. Nature. 2005; 435:1267-1270. https://doi. org/10.1038/nature03669. [PubMed]

26. Olshen A, Tang M, Cortes J, Gonen M, Hughes T, Branford S, Quintás-Cardama A, Michor F. Dynamics of chronic myeloid leukemia response to dasatinib, nilotinib, and highdose imatinib. Haematologica. 2014; 99:1701-1709. https:// doi.org/10.3324/haematol.2013.085977. [PubMed]

27. White D, Saunders V, Lyons AB, Branford S, Grigg A, To LB, Hughes T. In vitro sensitivity to imatinibinduced inhibition of ABL kinase activity is predictive of molecular response in patients with de novo CML. Blood. 2005; 106:2520-2526. https://doi.org/10.1182/ blood-2005-03-1103. [PubMed]

28. Hai A, Kizilbash NA, Zaidi SHH, Alruwaili J, Shahzad K. Differences in structural elements of BcrAbl oncoprotein isoforms in Chronic Myelogenous Leukemia. Bioinformation. 2014; 10:108-114. https://doi. org/10.6026/97320630010108. [PubMed] 
29. Pagani IS, Dang P, Kommers IO, Goyne JM, Nicola M, Saunders VA, Braley J, White DL, Yeung DT, Branford S, Hughes TP, Ross DM. BCR-ABL1 genomic DNA PCR response kinetics during first-line imatinib treatment of chronic myeloid leukemia. Haematologica. 2018; 103:2026-2032. https://doi.org/10.3324/haematol.2018.189787. [PubMed]

30. Clark RE, Dodi IA, Hill SC, Lill JR, Aubert G, Macintyre AR, Rojas J, Bourdon A, Bonner PLR, Wang L, Christmas SE, Travers PJ, Creaser CS, et al. Direct evidence that leukemic cells present HLA-associated immunogenic peptides derived from the BCR-ABL b3a2 fusion protein. Blood. 2001; 98:2887-2893. https://doi.org/10.1182/blood. V98.10.2887. [PubMed]

31. Cross NCP, White HE, Colomer D, Ehrencrona H, Foroni L, Gottardi E, Lange T, Lion T, Machova Polakova K, Dulucq S, Martinelli G, Oppliger Leibundgut E, Pallisgaard $\mathrm{N}$, et al. Laboratory recommendations for scoring deep molecular responses following treatment for chronic myeloid leukemia. Leukemia. 2015; 29:999-1003. https:// doi.org/10.1038/leu.2015.29. [PubMed]

32. Baccarani M, Deininger MW, Rosti G, Hochhaus A, Soverini S, Apperley JF, Cervantes F, Clark RE, Cortes JE, Guilhot F, Hjorth-Hansen H, Hughes TP, Kantarjian $\mathrm{HM}$, et al. European LeukemiaNet recommendations for the management of chronic myeloid leukemia: 2013. Blood. 2013; 122:872-884. https://doi.org/10.1182/ blood-2013-05-501569. [PubMed]
33. Cross NCP, White HE, Müller MC, Saglio G, Hochhaus A. Standardized definitions of molecular response in chronic myeloid leukemia. Leukemia. 2012; 26:2172-2175. https:// doi.org/10.1038/leu.2012.104. [PubMed]

34. Damaj G, Delabesse E, Le Bihan C, Asnafi V, Rachid M, Lefrère F, Radford-Weiss I, Macintyre E, Hermine O, Varet B. Typical essential thrombocythaemia does not express bcrabelson fusion transcript. Br J Haematol. 2002; 116:812816. https://doi.org/10.1046/j.0007-1048.2002.03343.x. [PubMed]

35. Gabert J, Beillard E, van der Velden VHJ, Bi W, Grimwade D, Pallisgaard N, Barbany G, Cazzaniga G, Cayuela JM, Cavé H, Pane F, Aerts JLE, De Micheli D, et al. Standardization and quality control studies of "realtime" quantitative reverse transcriptase polymerase chain reaction of fusion gene transcripts for residual disease detection in leukemia - a Europe Against Cancer program. Leukemia. 2003; 17:2318-2357. https://doi.org/10.1038/ sj.leu.2403135. [PubMed] 